J. Korean Math. Soc. 51 (2014), No. 6, pp. 1155-1175

http://dx.doi.org/10.4134/JKMS.2014.51.6.1155

\title{
A UNIFIED CONVERGENCE ANALYSIS FOR SECANT-TYPE METHODS
}

\author{
Ioannis Konstantinos Argyros and Ángel Alberto Magreñán
}

\begin{abstract}
We present a unified local and semilocal convergence analysis for secant-type methods in order to approximate a locally unique solution of a nonlinear equation in a Banach space setting. Our analysis includes the computation of the bounds on the limit points of the majorizing sequences involved. Under the same computational cost our semilocal convergence criteria can be weaker; the error bounds more precise and in the local case the convergence balls can be larger and the error bounds tighter than in earlier studies such as $[1-3,7-14,16,20,21]$ at least for the cases of Newton's method and the secant method. Numerical examples are also presented to illustrate the theoretical results obtained in this study.
\end{abstract}

\section{Introduction}

In this study we are concerned with the problem of approximating a locally unique solution $x^{\star}$ of the nonlinear equation

$$
F(x)=0,
$$

where $F$ is a Fréchet-differentiable operator defined on a nonempty subset $\mathcal{D}$ of a Banach space $\mathcal{X}$ with values in a Banach space $\mathcal{Y}$. A lot of problems from Applied Sciences can be expressed in a form like (1.1) using mathematical modelling [4]. The solutions of these equations can be found in closed form only in special cases. That is why the most solution methods for these equations are iterative. The convergence analysis of iterative methods is usually divided into two categories: semilocal and local convergence analysis. In the semilocal convergence analysis one derives convergence criteria from the information around an initial point whereas in the local analysis one finds estimates of the radii of convergence balls from the information around a solution. If $\mathcal{X}=\mathcal{Y}$ and $Q(x)=F(x)+x$, then the solution $x^{*}$ of equation (1.1) is very important in fixed point theory.

Received November 16, 2013; Revised March 13, 2014.

2010 Mathematics Subject Classification. 65H10, 65G99, 65B05, 65N30, 47H17, 49M15.

Key words and phrases. secant-type method, Banach space, majorizing sequence, divided difference, local convergence, semilocal convergence.

The research is supported by the grant MTM2011-28636-C02-01. 
We study the convergence of the secant-type method

$$
x_{n+1}=x_{n}-\mathcal{A}_{n}^{-1} F\left(x_{n}\right), \mathcal{A}_{n}=\delta F\left(x_{n}, y_{n}\right) \text { for each } n=1,2, \ldots,
$$

where $x_{-1}, x_{0}$ are initial points, $y_{n}=\theta_{n} x_{n}+\left(1-\theta_{n}\right) x_{n-1}, \theta_{n} \in \mathbb{R}$. Here $\mathcal{A}_{n} \in \mathcal{L}(\mathcal{X}, \mathcal{Y}), x, y \in \mathcal{D}$ is a consistent approximation of the Fréchet-derivative of $F$. (See page 182 of [14] or the second estimate in condition $\left(\mathcal{D}_{4}\right)$ of Definition 3.1.) $\mathcal{L}(\mathcal{X}, \mathcal{Y})$ stands for the space of bounded linear operators from $\mathcal{X}$ to $\mathcal{Y}$. Many iterative methods are special cases of (1.2). Indeed, if $\theta_{n}=1$, then we obtain Newton's method

$$
x_{n+1}=x_{n}-F^{\prime}\left(x_{n}\right)^{-1} F\left(x_{n}\right) \text { for each } n=0,1,2, \ldots ;
$$

if $\theta_{n}=0$, we obtain the secant method

$$
x_{n+1}=x_{n}-\delta F\left(x_{n}, x_{n-1}\right)^{-1} F\left(x_{n}\right) \text { for each } n=0,1,2, \ldots ;
$$

if $\theta_{n}=2$, we obtain the Kurchatov method

$$
x_{n+1}=x_{n}-\delta F\left(x_{n}, 2 x_{n}-x_{n-1}\right)^{-1} F\left(x_{n}\right) \text { for each } n=0,1,2, \ldots
$$

Other choices of $\theta_{n}$ are also possible $[1,2,5,7,10,11,13,14,20,21]$. There is a plethora of sufficient convergence criteria for special cases of secant-type methods (1.3)-(1.5) under Lipschitz-type conditions (1.2) (see [1-11,13-21] and the references there in). Therefore, it is important to study the convergence of the secant-type method in a unified way. It is interesting to notice that although we use very general majorizing sequences for $\left\{x_{n}\right\}$ our technique leads in the semilocal case to: weaker sufficient convergence criteria; more precise estimates on the distances $\left\|x_{n}-x_{n-1}\right\|,\left\|x_{n}-x^{*}\right\|$ and an at least as precise information on the location of the solution $x^{*}$ in many interesting special cases such as Newton's method or the secant method (see Remark 3.3 and the Examples). Moreover, in the local case: a larger radius of convergence and more precise error estimates than in earlier studies such as $[7-9,11,13-21]$ are obtained in this study (see Remark 4.2 and the Examples).

The paper is organized as follows. In Section 2 we study the convergence of the majorizing sequences for $\left\{x_{n}\right\}$. Section 3 contains the semilocal and Section 4 the local convergence analysis for $\left\{x_{n}\right\}$. The numerical examples are given in the concluding Section 5. In particular, in the local case we present an example where the radius of convergence is larger than the one given by Rheinboldt [17] and Traub [19] for Newton's method. Moreover, in the semilocal case we provide an example involving a nonlinear integral equation of Chandrasekhar type [6] appearing in radiative transfer as well as an example involving a two point boundary value problem.

\section{Majorizing sequences for the secant-type method}

In this section, we shall first study some scalar sequences which are related to the secant-type method. 
Let there be parameters $c \geq 0, \nu \geq 0, \lambda \geq 0, \mu \geq 1, l_{0}>0$ and $l>0$ with $l_{0} \leq l$. Define the scalar sequence $\left\{\alpha_{n}\right\}$ by

$$
\left\{\begin{array}{c}
\alpha_{-1}=0, \alpha_{0}=c, \alpha_{1}=c+\nu \\
\alpha_{n+2}=\alpha_{n+1}+\frac{l\left(\alpha_{n+1}-\alpha_{n}+\lambda\left(\alpha_{n}-\alpha_{n-1}\right)\right)\left(\alpha_{n+1}-\alpha_{n}\right)}{1-l_{0}\left[\mu\left(\alpha_{n+1}-c\right)+\lambda\left(\alpha_{n}-c\right)+c\right]} \\
\quad \text { for each } n=0,1,2, \ldots
\end{array}\right.
$$

Special cases of the sequence $\left\{\alpha_{n}\right\}$ have been used as majorizing sequences for secant-type method by several authors. For example: Case 1 (secant method) $l_{0}=l, \lambda=1$ and $\mu=1$ has been studied in $[5,7,10,11,13,14,18,20]$ and for $l_{0} \leq l$ in $[2,3]$. Case 2 (Newton's method) $l_{0}=l, \lambda=0, c=0$ and $\mu=2$ has been studied in $[1,7-9,11,13,14,16,17,19-21]$ and for $l_{0} \leq l$ in $[2-4]$. In the present paper we shall study the convergence of sequence $\left\{\alpha_{n}\right\}$ by first simplifying it. Indeed, the purpose of the following transformations is to study the sequence (2.1) after using easier to study sequences defined by (2.3), (2.6) and (2.8). Let

$$
L_{0}=\frac{l_{0}}{1+(\mu+\lambda-1) l_{0} c} \text { and } L=\frac{l}{1+(\mu+\lambda-1) l_{0} c} .
$$

Using (2.1) and (2.2), sequence $\left\{\alpha_{n}\right\}$ can be written as

$$
\left\{\begin{array}{c}
\alpha_{-1}=0, \alpha_{0}=c, \alpha_{1}=c+\nu \\
\alpha_{n+2}=\alpha_{n+1}+\frac{L\left(\alpha_{n+1}-\alpha_{n}+\lambda\left(\alpha_{n}-\alpha_{n-1}\right)\right)\left(\alpha_{n+1}-\alpha_{n}\right)}{1-L_{0}\left(\mu \alpha_{n+1}+\lambda \alpha_{n}\right)} \\
\quad \text { for each } n=0,1,2, \ldots
\end{array}\right.
$$

Moreover, let

$$
L=b L_{0} \text { for some } b \geq 1
$$

and

$$
\beta_{n}=L_{0} \alpha_{n}
$$

Then, we can define sequence $\left\{\beta_{n}\right\}$ by

$$
\left\{\begin{array}{c}
\beta_{-1}=0, \beta_{0}=L_{0} c, \beta_{1}=L_{0}(c+\nu) \\
\beta_{n+2}=\beta_{n+1}+\frac{b\left(\beta_{n+1}-\beta_{n}+\lambda\left(\beta_{n}-\beta_{n-1}\right)\right)\left(\beta_{n+1}-\beta_{n}\right)}{1-\left(\mu \beta_{n+1}+\lambda \beta_{n}\right)} \\
\quad \text { for each } n=0,1,2, \ldots
\end{array}\right.
$$

Furthermore, let

$$
\gamma_{n}=\frac{1}{\mu+\lambda}-\beta_{n} \text { for each } n=0,1,2, \ldots
$$


Then, sequence $\left\{\gamma_{n}\right\}$ is defined by

$$
\left\{\begin{array}{c}
\gamma_{-1}=\frac{1}{\mu+\lambda}, \gamma_{0}=\frac{1}{\mu+\lambda}-L_{0} c, \gamma_{1}=\frac{1}{\mu+\lambda}-L_{0}(c+\nu) \\
\gamma_{n+2}=\gamma_{n+1}-\frac{b\left(\gamma_{n+1}-\gamma_{n}+\lambda\left(\gamma_{n}-\gamma_{n-1}\right)\right)\left(\gamma_{n+1}-\gamma_{n}\right)}{\mu \gamma_{n+1}+\lambda \gamma_{n}} \\
\quad \text { for each } n=0,1,2, \ldots
\end{array}\right.
$$

Finally, let

$$
\delta_{n}=1-\frac{\gamma_{n}}{\gamma_{n-1}} \text { for each } n=0,1,2, \ldots
$$

Then, we define the sequence $\left\{\delta_{n}\right\}$ by

$$
\left\{\begin{array}{c}
\delta_{0}=1-\frac{\gamma_{0}}{\gamma_{-1}}, \delta_{1}=1-\frac{\gamma_{1}}{\gamma_{0}} \\
\delta_{n+2}=\frac{b \delta_{n+1}\left(\lambda \delta_{n}+\left(1-\delta_{n}\right) \delta_{n+1}\right)}{\left(1-\delta_{n}\right)\left(1-\delta_{n+1}\right)\left(\mu\left(1-\delta_{n+1}\right)+\lambda\right)} \\
\quad \text { for each } n=0,1,2, \ldots
\end{array}\right.
$$

It is convenient for the study of the convergence of the sequence $\left\{\alpha_{n}\right\}$ to define polynomial $p$ by

$$
p(t)=\mu t^{3}-(\lambda+3 \mu+b) t^{2}+(2 \lambda+3 \mu+b(\lambda+1)) t-(\mu+\lambda) .
$$

We have that $p(0)=-(\mu+\lambda)<0$ and $p(1)=b \lambda>0$ for $\lambda>0$. It follows from the intermediate value theorem that $p$ has roots in $(0,1)$. Denote the smallest root by $\delta$. If $\lambda=0$, then $p(t)=(t-1)\left(\mu t^{2}-(2 \mu+b) t+\mu\right)$. Hence, we can choose the smallest root of $p$ given by $\frac{2 \mu+b-\sqrt{b^{2}+4 \mu b}}{2 \mu} \in(0,1)$ to be $\delta$ in this case. Note that in particular for Newton's method and secant method, respectively, we have that

$$
p(t)=(t-1)\left(2 t^{2}-(b+4) t+2\right)
$$

and

$$
p(t)=(t-2)\left(t^{2}-(b+2) t+1\right) .
$$

Hence, we obtain, respectively that

$$
\delta=\frac{4}{b+4+\sqrt{b^{2}+8 b}}
$$

and

$$
\delta=\frac{2}{b+2+\sqrt{b^{2}+4 b}} .
$$

Notice also that

$$
p(t) \leq 0 \text { for each } t \in(-\infty, \delta] .
$$

Next, we study the convergence of these sequences starting from $\left\{\delta_{n}\right\}$. 
Lemma 2.1. Let $\delta_{1}>0, \delta_{2}>0$ and $b \geq 1$ be given parameters. Suppose that

$$
0<\delta_{2} \leq \delta_{1} \leq \delta
$$

where $\delta$ was defined in (2.11). Let $\left\{\delta_{n}\right\}$ be the scalar sequence defined by (2.10). Then, the following assertions hold:

$\left(\mathcal{A}_{1}\right)$ If

$$
\delta_{1}=\delta_{2}
$$

$$
\delta_{n}=\delta \text { for each } n=1,2,3, \ldots
$$

$\left(\mathcal{A}_{2}\right)$ If

$$
0<\delta_{2}<\delta_{1}<\delta
$$

then sequence $\left\{\delta_{n}\right\}$ is decreasing and converges to 0 .

Proof. It follows from (2.10) and $\delta_{2} \leq \delta_{1}$ that $\delta_{3}>0$. We shall show that

$$
\delta_{3} \leq \delta_{2} \text {. }
$$

In view of (2.10) for $n=1$, it suffices to show that

(2.20) $p_{1}\left(\delta_{2}\right)=\mu\left(1-\delta_{1}\right) \delta_{2}^{2}-\left(1-\delta_{1}\right)(2 \mu+\lambda+b) \delta_{2}-(\mu+(1+b) \lambda) \delta_{1}+\mu+\lambda \geq 0$.

The discriminant $\Delta$ of the quadratic polynomial $p_{1}$ is given by

$$
\Delta=\left(1-\delta_{1}\right)\left[\left(1-\delta_{1}\right)\left(\lambda^{2}+2(2 \mu+\lambda) b+b^{2}\right)+4 \mu \lambda b \delta_{1}\right]>0 .
$$

Hence, $p_{1}$ has two distinct roots $\delta_{s}$ and $\delta_{l}$ with $\delta_{s}<\delta_{l}$. Polynomial $p_{1}$ is quadratic with respect to $\delta_{2}$ and the leading coefficient $\left(\mu\left(1-\delta_{1}\right)\right)$ is positive. Therefore, we have that

$$
p_{1}(t) \geq 0 \text { for each } t \in\left(-\infty, \delta_{s}\right] \cup\left[\delta_{l},+\infty\right)
$$

and

Then, (2.20) shall be true, if

$$
p_{1}(t) \leq 0 \text { for each } t \in\left[\delta_{s}, \delta_{l}\right] .
$$

$$
\delta_{2} \leq \delta_{s}
$$

By hypothesis (2.15) we have $\delta_{1} \leq \delta_{0}$. Then by (2.14) we get that $p\left(\delta_{1}\right) \leq 0 \Rightarrow$ $\delta_{1} \leq \delta_{s} \Rightarrow(2.22)$, since $\delta_{2} \leq \delta_{1}$ by hypothesis (2.15). Hence, we showed (2.19). Therefore, relation

$$
0<\delta_{k+1}<\delta_{k},
$$

holds for $k=2$. Then, we must show that

$$
0<\delta_{k+2}<\delta_{k+1} .
$$

It follow from (2.10), $\delta_{k}<1$ and $\delta_{k+1}<1$ that $\delta_{k+2}>0$. Then, in view of (2.10) the right hand side of (2.24) is true, if

$$
\frac{b \delta_{k+1}\left[\lambda \delta_{k}+\left(1-\delta_{k}\right) \delta_{k+1}\right]}{\left(1-\delta_{k}\right)\left(1-\delta_{k+1}\right)\left[\lambda+\mu\left(1-\delta_{k+1}\right)\right]} \leq \delta_{k+1}
$$


or

$$
p\left(\delta_{k}\right) \leq 0
$$

which is true by (2.14) since $\delta_{k} \leq \delta_{1} \leq \delta$. The induction for (2.23) is complete. If $\delta_{1}=\delta_{2}=\delta$, then it follows from (2.10) for $n=1$ that $\delta_{3}=\delta$ and $\delta_{n}=\delta$ for $n=4,5, \ldots$, which shows (2.17). If $\delta_{2}<\delta_{1}$, the sequence $\left\{\delta_{n}\right\}$ is decreasing, bounded below by 0 and as such it converges to its unique largest lower bound denoted by $\gamma$. We then have from (2.10) that

$$
\gamma=\frac{b \gamma[\lambda \gamma+(1-\gamma) \gamma]}{(1-\gamma)^{2}[\lambda+\mu(1-\gamma)]} \Rightarrow \gamma=\delta \text { or } \gamma=0
$$

But $\gamma \leq \delta_{1} \leq \delta$. Hence, we conclude that $\gamma=0$.

Next, we present three results for the convergence of sequences $\left\{\alpha_{n}\right\},\left\{\beta_{n}\right\}$ and $\left\{\gamma_{n}\right\}$ under conditions that are not all the same with the ones in Lemma 2.1 (see e.g. (2.28)).

Lemma 2.2. Suppose that the hypothesis (2.18) is satisfied. Then the sequence $\left\{\gamma_{n}\right\}$ is decreasingly convergent and sequences $\left\{\alpha_{n}\right\}$ and $\left\{\beta_{n}\right\}$ are increasingly convergent. Moreover, the following estimate holds:

$$
l_{0} c<1 \text {. }
$$

Proof. Using (2.2) and (2.9) we get that

$\gamma_{n}=\left(1-\delta_{n}\right) \gamma_{n-1}=\cdots=\left(1-\delta_{n}\right) \cdots\left(1-\delta_{1}\right) \gamma_{0}=\left(1-\delta_{n}\right) \cdots\left(1-\delta_{1}\right) \gamma_{0}>0$.

In view of (2.18) we have in turn that

$$
\begin{aligned}
\delta_{1}>0 & \Rightarrow 1-\frac{\gamma_{1}}{\gamma_{0}}>0 \\
& \Rightarrow \quad \gamma_{0}=\frac{1-(\mu+\lambda) L_{0} c}{\mu+\lambda}>0 \\
& \Rightarrow \gamma_{0}=\frac{1-l_{0} c}{(\mu+\lambda)\left[1+(\mu+\lambda-1) l_{0} c\right]}>0 \\
& \Rightarrow(2.28)
\end{aligned}
$$

and by the preceding equation we deduce that $\gamma_{n}>0$ for each $n=1,2, \ldots$ and

$$
\gamma_{n}<\gamma_{n-1} \text { for each } n=1,2, \ldots \text {, }
$$

since $\delta_{n}<1$. Hence, sequence $\left\{\gamma_{n}\right\}$ converges to its unique largest lower bound denoted by $\gamma^{*}$. We also have that $\beta_{n}=\frac{1}{\mu+\lambda}-\gamma_{n}<\frac{1}{\mu+\lambda}$. Thus, the sequence $\left\{\beta_{n}\right\}$ is increasing, bounded from above by $\frac{1}{\mu+\lambda}$ and as such it converges to its unique least upper bound denoted by $\beta^{*}$. Then, in view of (2.5) sequence $\left\{\alpha_{n}\right\}$ is also increasing, bounded from above by $\frac{L_{0}^{-1}}{\mu+\lambda}$ and such it also converges to its unique least upper bound denoted by $\alpha^{*}$. 
Lemma 2.3. Suppose that (2.15) and (2.16) are satisfied. Then the following assertions hold for each $n=1,2, \ldots$

$$
\begin{gathered}
\delta_{n}=\delta, \\
\gamma_{n}=(1-\delta)^{n} \gamma_{0}, \gamma^{*}=\lim _{n \rightarrow \infty} \gamma_{n}=0, \\
\beta_{n}=\frac{1}{\mu+\lambda}-(1-\delta)^{n} \gamma_{0}, \beta^{*}=\lim _{n \rightarrow \infty} \beta_{n}=\frac{1}{\mu+\lambda}
\end{gathered}
$$

and

$$
\alpha_{n}=\frac{1}{L_{0}}\left[\frac{1}{\mu+\lambda}-(1-\delta)^{n} \gamma_{0}\right], \alpha^{*}=\lim _{n \rightarrow \infty} \alpha_{n}=\frac{1}{L_{0}(\mu+\lambda)} .
$$

Corollary 2.4. Suppose that the hypotheses of Lemma 2.1 and Lemma 2.2 hold. Then the sequence $\left\{\alpha_{n}\right\}$ defined in (2.1) is nondecreasing and converges to

$$
\alpha^{*}=\beta^{*} \frac{1+(\mu+\lambda-1) l_{0} c}{l_{0}} .
$$

Next, we present lower and upper bounds on the limit point $\alpha^{*}$.

Lemma 2.5. Suppose that the condition (2.18) is satisfied. Then the following assertion holds

$$
b_{1}^{1} \leq \alpha^{*} \leq b_{2}^{1}
$$

where

$$
b_{1}^{1}=\frac{1+(\mu+\lambda-1) l_{0} c}{l_{0}}\left[\frac{1}{\mu+\lambda}-\exp \left(-2\left(\frac{\delta_{1}}{2-\delta_{1}}+\frac{\delta_{2}}{2-\delta_{2}}\right)\right)\right],
$$

$$
\begin{gathered}
b_{2}^{1}=\frac{1+(\mu+\lambda-1) l_{0} c}{l_{0}}\left[\frac{1}{\mu+\lambda}-\exp \left(\delta^{*}\right)\right] \\
\delta^{*}=-\left[\frac{1}{1-\delta_{1}}\left(\delta_{1}+\frac{\delta_{2}}{1-r}\right)+\ln \left(\frac{(\mu+\lambda)\left(1-(\mu+\lambda-1) l_{0} c\right)}{1-l_{0} c}\right)\right]
\end{gathered}
$$

and

$$
r=b \frac{\lambda \delta_{1}+\delta_{2}\left(1-\delta_{1}\right)}{\left(1-\delta_{1}\right)\left(1-\delta_{2}\right)\left(\lambda+\mu\left(1-\delta_{2}\right)\right)} .
$$

Proof. Using (2.18) and (2.28) we have that $0<\delta_{3}<\delta_{2}<\delta_{1}$. Let us assume that $0<\delta_{k+1}<\delta_{k}<\cdots<\delta_{1}$. Then, it follows from the induction hypotheses and (2.30) that

$$
\begin{aligned}
\delta_{k+2} & =\delta_{k+1} b \frac{\delta_{k}+\delta_{k+1}\left(1-\delta_{k}\right)}{\left(1-\delta_{k}\right)\left(1-\delta_{k+1}\right)\left(2-\delta_{k+1}\right)} \\
& <r \delta_{k+1}<r^{2} \delta_{k} \leq \cdots \leq r^{k-1} \delta_{3} \leq r^{k} \delta_{2} .
\end{aligned}
$$

We have that

$$
\gamma^{*}=\lim _{n \rightarrow \infty} \gamma_{n}=\prod_{i=1}^{\infty}\left(1-\delta_{n}\right) \gamma_{0}
$$


This is equivalent to

$$
\ln \left(\frac{1}{\gamma^{*}}\right)=\sum_{n=1}^{\infty} \ln \left(\frac{1}{1-\delta_{n}}\right)+\ln \left(\frac{(\mu+\lambda)\left(1+(\mu+\lambda-1) l_{0} c\right)}{1-l_{0} c}\right),
$$

recalling that $\gamma_{0}=\left(1-l_{0} c\right) /\left((\mu+\lambda)\left(1+(\mu+\lambda-1) l_{0} c\right)\right)$. We shall use the following bounds for $\ln t, t>1$ :

$$
2\left(\frac{t-1}{t+1}\right) \leq \ln t \leq \frac{t^{2}-1}{2 t} .
$$

First, we shall find an upper bound for $\ln \left(1 / \gamma^{*}\right)$. We have that

$$
\begin{aligned}
& \ln \left(1 / \gamma^{*}\right) \\
\leq & \sum_{n=1}^{\infty} \frac{\delta_{n}\left(2-\delta_{n}\right)}{2\left(1-\delta_{n}\right)}+\ln \left(\frac{(\mu+\lambda)\left(1+(\mu+\lambda-1) l_{0} c\right)}{1-l_{0} c}\right) \\
\leq & \frac{1}{1-\delta_{1}} \sum_{n=1}^{\infty} \delta_{n}+\ln \left(\frac{(\mu+\lambda)\left(1+(\mu+\lambda-1) l_{0} c\right)}{1-l_{0} c}\right) \\
\leq & \frac{1}{1-\delta_{1}}\left(\delta_{1}+\delta_{2}+\delta_{3}+\cdots\right)+\ln \left(\frac{(\mu+\lambda)\left(1+(\mu+\lambda-1) l_{0} c\right)}{1-l_{0} c}\right) \\
\leq & \frac{1}{1-\delta_{1}}\left(\delta_{1}+\delta_{2}+r \delta_{2}+\cdots+r^{n} \delta_{2}+\cdots\right)+\ln \left(\frac{(\mu+\lambda)\left(1+(\mu+\lambda-1) l_{0} c\right)}{1-l_{0} c}\right) \\
\leq & \frac{1}{1-\delta_{1}}\left(\delta_{1}+\delta_{2}\left(r+r^{2}+\cdots+r^{n}+\cdots\right)+\ln \left(\frac{(\mu+\lambda)\left(1+(\mu+\lambda-1) l_{0} c\right)}{1-l_{0} c}\right)\right. \\
\leq & \frac{1}{1-\delta_{1}}\left(\delta_{1}+\frac{\delta_{2}}{1-r}\right)+\ln \left(\frac{(\mu+\lambda)\left(1+(\mu+\lambda-1) l_{0} c\right)}{1-l_{0} c}\right)=-\delta^{*} .
\end{aligned}
$$

As $\beta^{*}=1 /(\mu+\lambda)-\gamma^{*}$ and $\alpha^{*}=L_{0}^{-1} \beta^{*}$, we obtain the upper bound in (2.29). Moreover, in order to obtain the lower bound for $\ln \left(1 / \gamma^{*}\right)$, we have that

$$
\ln \left(1 / \gamma^{*}\right) \geq 2 \sum_{n=1}^{\infty} \frac{\delta_{n}}{2-\delta_{n}}>2\left(\frac{\delta_{1}}{2-\delta_{1}}+\frac{\delta_{2}}{2-\delta_{2}}\right)
$$

which implies the lower bound in (2.29).

From now on we shall denote by $\left(C^{1}\right)$ the hypothesis of Lemma 2.1 and Lemma 2.2.

Remark 2.6. (a) Let us introduce the notation

$$
c^{N}=\alpha_{N-1}-\alpha_{N-2}, \nu^{N}=\alpha_{N}-\alpha_{N-1}
$$

for some integer $N \geq 1$. Notice that $c^{1}=\alpha_{0}-\alpha_{-1}=c$ and $\nu^{1}=\alpha_{1}-\alpha_{0}=\nu$. The results in the preceding lemmas can be weakened even further as follows. Consider the convergence criteria $\left(C_{*}^{N}\right)$ for $N>1$ : $\left(C^{1}\right)$ with $c, \nu$ replaced by $c^{N}, \nu^{N}$, respectively

$$
\alpha_{-1}<\alpha_{0}<\alpha_{1}<\cdots<\alpha_{N}<\alpha_{N+1},
$$




$$
l_{0}\left[\mu\left(\alpha_{N+1}-c^{N}\right)+\lambda\left(\alpha_{N}-c^{N}\right)+c^{N}\right]<1 .
$$

Then, the preceding results hold with $c, \nu, \delta_{1}, \delta_{2}, b_{1}^{1}, b_{2}^{1}$ replaced, respectively, by $c^{N}, \nu^{N}, \delta_{N}, \delta_{N+1}, b_{1}^{N}, b_{2}^{N}$.

(b) Notice that if

$$
l_{0}\left[\mu\left(\alpha_{n+1}-c\right)+\lambda\left(\alpha_{n}-c\right)+c\right]<1 \text { holds for each } n=0,1,2, \ldots,
$$

then, it follows from (2.1) that sequence $\left\{\alpha_{n}\right\}$ is increasing, bounded from above by $\frac{1+(\mu+\lambda-1) l_{0} c}{l_{0}(\mu+\lambda)}$ and as such it converges to its unique least upper bound $\alpha^{*}$. Criterion (2.31) is the weakest of all the preceding convergence criteria for sequence $\left\{\alpha_{n}\right\}$. Clearly all the preceding criteria imply (2.31). Finally, define the criteria for $N \geq 1$

$$
\left(I^{N}\right)=\left\{\begin{array}{l}
\left(C_{*}^{N}\right) \\
(2.31) \text { if criteria }\left(C_{*}^{N}\right) \text { fail. }
\end{array}\right.
$$

Lemma 2.7. Suppose that the conditions (2.18) and (2.28) hold. Then the following assertion holds

$$
b_{1}^{1} \leq \alpha^{*} \leq b_{2}^{1}
$$

where

$$
\begin{gathered}
b_{1}^{1}=\frac{1+(\mu+\lambda-1) l_{0} c}{l_{0}}\left[\frac{1}{\mu+\lambda}-\exp \left(-2\left(\frac{\delta_{1}}{2-\delta_{1}}+\frac{\delta_{2}}{2-\delta_{2}}\right)\right)\right] \\
b_{2}^{1}=\frac{1+(\mu+\lambda-1) l_{0} c}{l_{0}}\left[\frac{1}{\mu+\lambda}-\exp \left(\delta^{*}\right)\right] \\
\delta^{*}=-\left[\frac{1}{1-\delta_{1}}\left(\delta_{1}+\frac{\delta_{2}}{1-r}\right)+\ln \left(\frac{(\mu+\lambda)\left(1-(\mu+\lambda-1) l_{0} c\right)}{1-l_{0} c}\right)\right]
\end{gathered}
$$

and

$$
r=b \frac{\lambda \delta_{1}+\delta_{2}\left(1-\delta_{1}\right)}{\left(1-\delta_{1}\right)\left(1-\delta_{2}\right)\left(\lambda+\mu\left(1-\delta_{2}\right)\right)} .
$$

Proof. Using (2.18) and (2.28) we have that $0<\delta_{3}<\delta_{2}<\delta_{1}$. Let us assume that $0<\delta_{k+1}<\delta_{k}<\cdots<\delta_{1}$. Then, it follows from the induction hypotheses and (2.34) that

$$
\begin{aligned}
\delta_{k+2} & =\delta_{k+1} b \frac{\delta_{k}+\delta_{k+1}\left(1-\delta_{k}\right)}{\left(1-\delta_{k}\right)\left(1-\delta_{k+1}\right)\left(2-\delta_{k+1}\right)} \\
& <r \delta_{k+1}<r^{2} \delta_{k} \leq \cdots \leq r^{k-1} \delta_{3} \leq r^{k} \delta_{2} .
\end{aligned}
$$

We have that

$$
\gamma^{*}=\lim _{n \rightarrow \infty} \gamma_{n}=\prod_{i=1}^{\infty}\left(1-\delta_{n}\right) \gamma_{0}
$$

This is equivalent to

$$
\ln \left(\frac{1}{\gamma^{*}}\right)=\sum_{n=1}^{\infty} \ln \left(\frac{1}{1-\delta_{n}}\right)+\ln \left(\frac{(\mu+\lambda)\left(1+(\mu+\lambda-1) l_{0} c\right)}{1-l_{0} c}\right),
$$


recalling that $\gamma_{0}=\left(1-l_{0} c\right) /\left((\mu+\lambda)\left(1+(\mu+\lambda-1) l_{0} c\right)\right)$. We shall use the following bounds for $\ln t, t>1$ :

$$
2\left(\frac{t-1}{t+1}\right) \leq \ln t \leq \frac{t^{2}-1}{2 t} .
$$

First, we shall find an upper bound for $\ln \left(1 / \gamma^{*}\right)$. We have that

$$
\begin{aligned}
& \ln \left(1 / \gamma^{*}\right) \\
\leq & \sum_{n=1}^{\infty} \frac{\delta_{n}\left(2-\delta_{n}\right)}{2\left(1-\delta_{n}\right)}+\ln \left(\frac{(\mu+\lambda)\left(1+(\mu+\lambda-1) l_{0} c\right)}{1-l_{0} c}\right) \\
\leq & \frac{1}{1-\delta_{1}} \sum_{n=1}^{\infty} \delta_{n}+\ln \left(\frac{(\mu+\lambda)\left(1+(\mu+\lambda-1) l_{0} c\right)}{1-l_{0} c}\right) \\
\leq & \frac{1}{1-\delta_{1}}\left(\delta_{1}+\delta_{2}+\delta_{3}+\cdots\right)+\ln \left(\frac{(\mu+\lambda)\left(1+(\mu+\lambda-1) l_{0} c\right)}{1-l_{0} c}\right) \\
\leq & \frac{1}{1-\delta_{1}}\left(\delta_{1}+\delta_{2}+r \delta_{2}+\cdots+r^{n} \delta_{2}+\cdots\right)+\ln \left(\frac{(\mu+\lambda)\left(1+(\mu+\lambda-1) l_{0} c\right)}{1-l_{0} c}\right) \\
\leq & \frac{1}{1-\delta_{1}}\left(\delta_{1}+\delta_{2}\left(r+r^{2}+\cdots+r^{n}+\cdots\right)+\ln \left(\frac{(\mu+\lambda)\left(1+(\mu+\lambda-1) l_{0} c\right)}{1-l_{0} c}\right)\right. \\
\leq & \frac{1}{1-\delta_{1}}\left(\delta_{1}+\frac{\delta_{2}}{1-r}\right)+\ln \left(\frac{(\mu+\lambda)\left(1+(\mu+\lambda-1) l_{0} c\right)}{1-l_{0} c}\right)=-\delta^{*} .
\end{aligned}
$$

As $\beta^{*}=1 /(\mu+\lambda)-\gamma^{*}$ and $\alpha^{*}=L_{0}^{-1} \beta^{*}$, we obtain the upper bound in (2.34). Moreover, in order to obtain the lower bound for $\ln \left(1 / \gamma^{*}\right)$, we have that

$$
\ln \left(1 / \gamma^{*}\right) \geq 2 \sum_{n=1}^{\infty} \frac{\delta_{n}}{2-\delta_{n}}>2\left(\frac{\delta_{1}}{2-\delta_{1}}+\frac{\delta_{2}}{2-\delta_{2}}\right)
$$

which implies the lower bound in (2.34).

\section{Semilocal convergence of the secant-type method}

In this section, we first present the semilocal convergence of the secant-type method using $\left\{\alpha_{n}\right\}$ (defined in (2.1)) as a majorizing sequence. Let $U(x, R)$ stand for an open ball centered at $x \in X$ with radius $R>0$. Let $\bar{U}(x, R)$ denote its closure. We shall study the secant method for triplets $\left(\mathcal{F}, x_{-1}, x_{0}\right)$ belonging to the class $\mathcal{K}=\mathcal{K}\left(l_{0}, l, \nu, c, \lambda, \mu\right)$ defined as follows.

Definition 3.1. Let $l_{0}, l, \nu, c, \lambda, \mu$ be constants satisfying the hypotheses $\left(I^{N}\right)$ for some fixed integer $N \geq 1$. A triplet $\left(\mathcal{F}, x_{-1}, x_{0}\right)$ belongs to the class $\mathcal{K}=$ $\mathcal{K}\left(l_{0}, l, \nu, c, \lambda, \mu\right)$ if:

$\left(\mathcal{D}_{1}\right) \mathcal{F}$ is a nonlinear operator defined on a convex subset $D$ of a Banach space $\mathcal{X}$ with values in a Banach space $\mathcal{Y}$. 
$\left(\mathcal{D}_{2}\right) x_{-1}$ and $x_{0}$ are two points belonging to the interior $D^{0}$ of $D$ and satisfying the inequality

$$
\left\|x_{0}-x_{-1}\right\| \leq c .
$$

$\left(\mathcal{D}_{3}\right)$ There exist a sequence $\left\{\theta_{n}\right\}$ of real numbers and $\lambda, \mu$ such that $\left|1-\theta_{n}\right| \leq$ $\lambda$ and $1+\left|\theta_{n}\right| \leq \mu$ for each $n=0,1,2, \ldots$.

$\left(\mathcal{D}_{4}\right) \mathcal{F}$ is Fréchet-differentiable on $D^{0}$ and there exists an operator $\delta \mathcal{F}$ : $\mathcal{D}^{0} \times D^{0} \rightarrow \mathrm{E}(X, Y)$ such that $\mathcal{A}^{-1}=\delta \mathcal{F}\left(x_{0}, y_{0}\right)^{-1} \in \mathrm{E}(Y, X)$ for all $x, y, z \in D$ then, the following hold

$$
\begin{gathered}
\left\|\mathcal{A}^{-1} \mathcal{F}\left(x_{0}\right)\right\| \leq \nu \\
\left\|\mathcal{A}^{-1}\left(\delta \mathcal{F}(x, y)-\mathcal{F}^{\prime}(z)\right)\right\| \leq l(\|x-z\|+\|y-z\|)
\end{gathered}
$$

and

$$
\left\|\mathcal{A}^{-1}\left(\delta \mathcal{F}(x, y)-\mathcal{F}^{\prime}\left(x_{0}\right)\right)\right\| \leq l_{0}\left(\left\|x-x_{0}\right\|+\left\|y-x_{0}\right\|\right),
$$

where $y_{0}=\theta_{0} x_{0}+\left(1-\theta_{0}\right) x_{-1}$.

$\left(\mathcal{D}_{5}\right)$

$$
\bar{U}\left(x_{0}, \alpha_{0}^{*}\right) \subseteq D_{c}=\{x \in D: \mathcal{F} \text { is continuous at } x\} \subseteq D,
$$

where $\alpha_{0}^{*}=(\mu+\lambda-1)\left(\alpha^{*}-c\right)$ and $\alpha^{*}$ is given in Lemma 2.3.

Next, we present the semilocal convergence result for the secant method.

Theorem 3.2. If $\left(\mathcal{F}, x_{-1}, x_{0}\right) \in \mathcal{K}\left(l_{0}, l, \nu, c, \lambda, \mu\right)$, then the sequence $\left\{x_{n}\right\}(n \geq$ $-1)$ generated by the secant-type method is well defined, remains in $\bar{U}\left(x_{0}, \alpha_{0}^{*}\right)$ for each $n=0,1,2, \ldots$ and converges to a unique solution $x^{*} \in \bar{U}\left(x_{0}, \alpha^{*}-c\right)$ of (1.1). Moreover, the following assertions hold for each $n=0,1,2, \ldots$

$$
\left\|x_{n}-x_{n-1}\right\| \leq \alpha_{n}-\alpha_{n-1}
$$

$$
\left\|x^{*}-x_{n}\right\| \leq \alpha^{*}-\alpha_{n},
$$

where sequence $\left\{\alpha_{n}\right\}(n \geq 0)$ is given in (2.1). Furthermore, if there exists $R$ such that

(3.3) $\bar{U}\left(x_{0}, R\right) \subseteq D, R \geq \alpha^{*}-c$ and $l_{0}\left(\alpha^{*}-c+R\right)+\left\|\mathcal{A}^{-1}\left(\mathcal{F}^{-1}\left(x_{0}\right)-\mathcal{A}\right)\right\|<1$, then the solution $x^{*}$ is unique in $\bar{U}\left(x_{0}, R\right)$.

Proof. First, we show that $\mathcal{M}=\delta \mathcal{F}\left(x_{k+1}, y_{k+1}\right)$ is invertible for $x_{k+1}, y_{k+1} \in$ $\bar{U}\left(x_{0}, \alpha_{0}^{*}\right)$. By $\left(\mathcal{D}_{2}\right),\left(\mathcal{D}_{3}\right)$ and $\left(\mathcal{D}_{4}\right)$, we have that

$$
\begin{aligned}
\left\|y_{k+1}-x_{0}\right\| & \leq\left\|\theta_{k}\left(x_{k+1}-x_{0}\right)+\left(1-\theta_{k+1}\right)\left(x_{k}-x_{0}\right)\right\| \\
& \leq\left|\theta_{k+1}\right|\left\|x_{k+1}-x_{0}\right\|+\left|1-\theta_{k+1}\right|\left\|x_{k}-x_{0}\right\| \\
& \leq(\mu-1)\left(\alpha^{*}-c\right)+\lambda\left(\alpha^{*}-c\right)=\alpha_{0}^{*}
\end{aligned}
$$

and

$$
\begin{gathered}
\\
\left\|I-\mathcal{A}^{-1} \mathcal{M}\right\| \\
=\left\|\mathcal{A}^{-1}(\mathcal{M}-\mathcal{A})\right\|
\end{gathered}
$$




$$
\begin{aligned}
& \leq\left\|\mathcal{A}^{-1}\left(\mathcal{M}-\mathcal{F}^{\prime}\left(x_{0}\right)\right)\right\|+\left\|\mathcal{A}^{-1}\left(\mathcal{F}^{\prime}\left(x_{0}\right)-\mathcal{A}\right)\right\| \\
& \leq l_{0}\left(\left\|x_{k+1}-x_{0}\right\|+\left\|y_{k+1}-x_{0}\right\|+\left\|x_{0}-x_{-1}\right\|\right) \\
& \leq l_{0}\left(\left\|x_{k+1}-x_{0}\right\|+\left|\theta_{k+1}\right|\left\|x_{k+1}-x_{0}\right\|+\left|1+\theta_{k+1}\right|\left\|x_{k+1}-x_{0}\right\|+c\right) \\
& \leq l_{0}\left(\mu\left(\alpha_{k+1}-c\right)+\lambda\left(\alpha_{k+1}-c\right)+c\right)<1 .
\end{aligned}
$$

Using the Banach lemma on invertible operators [8], [10], [14], [17], [18] and (3.4), we deduce that $\mathcal{M}$ is invertible and

$$
\left\|\mathcal{M}^{-1} \mathcal{A}\right\| \leq\left(1-l_{0}\left(\mu\left(\alpha_{k+1}-c\right)+\lambda\left(\alpha_{k+1}-c\right)+c\right)\right)^{-1} .
$$

By $\left(\mathcal{D}_{4}\right)$, we have

$$
\left\|\mathcal{A}^{-1}\left(\mathcal{F}^{\prime}(u)-\mathcal{F}^{\prime}(v)\right)\right\| \leq 2 l\|u-v\|, u, v \in D^{0} .
$$

We can write the identity

$$
\mathcal{F}^{\prime}(x)-\mathcal{F}^{\prime}(y)=\int_{0}^{1} \mathcal{F}^{\prime}(y+t(x-y)) d t(x-y) .
$$

Then, for all $x, y, u, v \in D^{0}$, we obtain

$$
\left\|\mathcal{A}^{-1}\left(\mathcal{F}(x)-\mathcal{F}(y)-\mathcal{F}^{\prime}(u)(x-y)\right)\right\| \leq l(\|x-u\|+\|y-u\|)\|x-y\|
$$

and

(3.9)

$\left\|\mathcal{A}^{-1}(\mathcal{F}(x)-\mathcal{F}(y)-\delta \mathcal{F}(u, v)(x-y))\right\| \leq l(\|x-v\|+\|y-v\|+\|u-v\|)\|x-y\|$.

By a continuity argument (3.6)-(3.9) remain valid if $x$ and/or $y$ belong to $D_{c}$. Next, we show (3.1). If (3.1) holds for all $n \leq k$ and if $\left\{x_{n}\right\}(n \geq 0)$ is well defined for $n=0,1,2, \ldots, k$, then

$$
\left\|x_{n}-x_{0}\right\| \leq \alpha_{n}-\alpha_{0}<\alpha^{*}-\alpha_{0}, n \leq k .
$$

That is (1.2) is well defined for $n=k+1$. For $n=-1$ and $n=0,(3.1)$ reduces to $\left\|x_{-1}-x_{0}\right\| \leq c$ and $\left\|x_{0}-x_{1}\right\| \leq \nu$. Suppose (3.1) holds for $n=-1,0,1, \ldots, k$ $(k \geq 0)$. By $(3.5),(3.9)$, and

$$
\mathcal{F}\left(x_{k+1}\right)=\mathcal{F}\left(x_{k+1}\right)-\mathcal{F}\left(x_{k}\right)-\mathcal{A}_{k}\left(x_{k+1} x_{k}\right)
$$

we obtain in turn the following estimates

$$
\begin{aligned}
& \left\|\mathcal{A}^{-1} \mathcal{F}\left(x_{k+1}\right)\right\| \\
= & \left\|\mathcal{A}^{-1}\left(\delta \mathcal{F}\left(x_{k+1}, x_{k}\right)-\mathcal{A}_{k}\right)\left(x_{k+1}-x_{k}\right)\right\| \\
\leq & \left(\left\|\mathcal{A}^{-1}\left(\delta \mathcal{F}\left(x_{k+1}, x_{k}\right)-\mathcal{F}^{\prime}\left(x_{k}\right)\right)\right\|+\left\|\mathcal{A}^{-1}\left(\mathcal{F}^{\prime}\left(x_{k}\right)-\mathcal{A}_{k}\right)\right\|\right)\left\|\left(x_{k+1}-x_{k}\right)\right\| \\
\leq & l\left[\left\|\left(x_{k+1}-x_{k}\right)\right\|+\left\|\left(x_{k}-y_{k}\right)\right\|\right]\left\|\left(x_{k+1}-x_{k}\right)\right\| \\
\leq & l\left(\alpha_{k+1}-\alpha_{k}+\left|1-\theta_{k}\right|\left(\alpha_{k}-\alpha_{k-1}\right)\left(\alpha_{k+1}-\alpha_{k}\right)\right)
\end{aligned}
$$

and

$$
\begin{gathered}
\left\|x_{k+2}-x_{k+1}\right\| \\
=\left\|\mathcal{A}_{k+1}^{-1} \mathcal{F}\left(x_{k+1}\right)\right\|
\end{gathered}
$$




$$
\begin{aligned}
& \leq\left\|\mathcal{A}_{k+1}^{-1} \mathcal{A}\right\|\left\|\mathcal{A}^{-1} \mathcal{F}\left(x_{k+1}\right)\right\| \\
& \leq \frac{l\left(\alpha_{k+1}-\alpha_{k}+\left|1-\theta_{k}\right|\left(\alpha_{k}-\alpha_{k-1}\right)\right)}{1-l_{0}\left[\left(1+\left|\theta_{k+1}\right|\right)\left(\alpha_{k+1}-c\right)+\left|1-\theta_{k+1}\right|\left(\alpha_{k}-c\right)+c\right]}\left(\alpha_{k+1}-\alpha_{k}\right) \\
& \leq \alpha_{k+2}-\alpha_{k+1} .
\end{aligned}
$$

The induction for (3.1) is complete. It follows from (3.1) and Lemma 2.1 that $\left\{x_{n}\right\}(n \geq-1)$ is a complete sequence in a Banach space $\mathcal{X}$ and as such it converges to some $x^{*} \in \bar{U}\left(x_{0}, \alpha^{*}-c\right)$ (since $\bar{U}\left(x_{0}, \alpha^{*}-c\right)$ is a closed set). By letting $k \rightarrow \infty$ in (3.12), we obtain $\mathcal{F}\left(x^{*}\right)=0$. Moreover, estimate (3.2) follows from (3.1) by using standard majoration techniques $[7,11,13]$. Finally, to show the uniqueness in $\bar{U}\left(x_{0}, R\right)$, let $y^{*} \in \bar{U}\left(x_{0}, R\right)$ be a solution (1.1). Set

$$
\mathcal{T}=\int_{0}^{1} \mathcal{F}^{\prime}\left(y^{*}+t\left(y^{*}-x^{*}\right)\right) d t .
$$

Using $\left(\mathcal{D}_{4}\right)$ and $(3.3)$ we get in turn that

$$
\begin{aligned}
\left\|\mathcal{A}^{-1}(\mathcal{A}-\mathcal{T})\right\| & =l_{0}\left(\left\|y^{*}-x_{0}\right\|+\left\|x^{*}-x_{0}\right\|\right)+\left\|\mathcal{A}^{-1}\left(\mathcal{F}^{\prime}\left(x_{0}\right)-\mathcal{A}\right)\right\| \\
& \leq l_{0}\left[\left(\alpha^{*}-\alpha_{0}\right)+R\right]+\left\|\mathcal{A}^{-1}\left(\mathcal{F}^{\prime}\left(x_{0}\right)-\mathcal{A}\right)\right\|<1
\end{aligned}
$$

If follows from (3.13) and the Banach lemma on invertible operators that $\mathcal{T}^{-1}$ exists. Using the identity:

$$
\mathcal{F}\left(x^{*}\right)-\mathcal{F}^{\prime}\left(y^{*}\right)=\mathcal{T}\left(x^{*}-y^{*}\right),
$$

we deduce that $x^{*}=y^{*}$.

Remark 3.3. If follows from the proof of Theorem 3.2 that sequences $\left\{r_{n}\right\}$, $\left\{s_{n}\right\}$ defined by

$$
\left\{\begin{array}{l}
r_{-1}=0, r_{0}=c, r_{1}=c+\nu \\
r_{2}=r_{1}+\frac{l_{0}\left(r_{1}-r_{0}+\left|1-\theta_{0}\right|\left(r_{0}-r_{-1}\right)\right)\left(r_{1}-r_{0}\right)}{1-l_{0}\left(\left(1+\left|\theta_{1}\right|\right)\left(r_{1}-r_{0}\right)\right)} \\
r_{n+2}=r_{n+1}+\frac{l\left(r_{n+1}-r_{n}+\left|1-\theta_{n}\right|\left(r_{n}-r_{n-1}\right)\right)\left(r_{n+1}-r_{n}\right)}{1-l_{0}\left[\left(1+\left|\theta_{n+1}\right|\right)\left(r_{n+1}-r_{0}\right)+\left(\left|1-\theta_{n+1}\right|\right)\left(r_{n}-r_{0}\right)+c\right]}
\end{array}\right.
$$

and

$$
\left\{\begin{array}{l}
s_{-1}=0, s_{0}=c, s_{1}=c+\nu \\
s_{2}=s_{1}+\frac{l_{0}\left(s_{1}-s_{0}+\lambda\left(s_{0}-s_{-1}\right)\right)\left(s_{1}-s_{0}\right)}{1-l_{0}\left(1+\left|\theta_{1}\right|\right)\left(s_{1}-s_{0}\right)} \\
s_{n+2}=s_{n+1}+\frac{l\left(s_{n+1}-s_{n}+\lambda\left(s_{n}-s_{n-1}\right)\right)\left(s_{n+1}-s_{n}\right)}{1-l_{0}\left(\mu\left(s_{n+1}-s_{0}\right)+\lambda\left(s_{n}-s_{0}\right)\right)+c}
\end{array}\right.
$$

respectively are more precise majorizing sequences for $\left\{x_{n}\right\}$. Clearly, these sequences also converge under the $\left(I^{N}\right)$ hypotheses.

A simple inductive argument shows that if $l_{0}<l$ for each $n=2,3, \ldots$

$$
\begin{gathered}
r_{n}<s_{n}<\alpha_{n} \\
r_{n+1}-r_{n}<s_{n+1}-s_{n}<\alpha_{n+1}-\alpha_{n}
\end{gathered}
$$


and

$$
r^{*}=\lim _{n \rightarrow \infty} r_{n} \leq s^{*}=\lim _{n \rightarrow \infty} s_{n} \leq \alpha^{*}=\lim _{n \rightarrow \infty} \alpha_{n} .
$$

In practice, one must choose $\left\{\theta_{n}\right\}$ so that the best error bounds are obtained (see also Section 4). Note also that sequences $\left\{r_{n}\right\}$ or $\left\{s_{n}\right\}$ may converge under even weaker hypotheses. The sufficient convergence criterion (2.15) determines the smallness of $c$ and $r$. This criterion can be solved for $c$ and $r$ (see for example the $h$ criteria or (3.29) that follow). Indeed, let us demonstrate the advantages in two popular cases:

Case 1: Newton's method (i.e., if $c=0, \lambda=0, \mu=1$ ). Then it can easily be seen that $\left\{s_{n}\right\}$ (and consequently $\left\{r_{n}\right\}$ ) converges provided that (see also [4])

$$
h_{2}=l_{2} \nu \leq 1
$$

where

$$
l_{2}=\frac{1}{4}\left(4 \kappa_{0}+\sqrt{\kappa_{0} \kappa}+\sqrt{\kappa_{0} \kappa+8 \kappa_{0}^{2}}\right),
$$

whereas sequence $\left\{x_{n}\right\}$ converges, if

$$
h_{1}=l_{1} \nu \leq 1
$$

where

$$
l_{1}=\frac{1}{4}\left(4 \kappa_{0}+\kappa+\sqrt{\kappa_{0}^{2}+8 \kappa \kappa_{0}}\right) .
$$

In the case $\kappa_{0}=\kappa$ (i.e., $b=1$ ), we obtain the famous for its simplicity and clarity Kantorovich sufficient convergent criteria [2] given by

$$
h=2 \kappa \nu \leq 1 .
$$

Notice however that

$$
h \leq 1 \Rightarrow h_{1} \leq 1 \Rightarrow h_{2} \leq 1
$$

but not necessarily vice versa unless if $\kappa_{0}=\kappa$. Moreover, we have that

$$
\frac{h_{1}}{h} \rightarrow \frac{1}{4}, \frac{h_{1}}{h} \rightarrow 0, \frac{h_{2}}{h_{1}} \rightarrow 0 \text { as } \frac{\kappa_{0}}{\kappa} \rightarrow 0 .
$$

Case 2: Secant method (i.e., for $\theta_{n}=0$ ). Schmidt [18], Potra-Ptáck [14], Dennis [7], Ezquerro el at. [10], used the majorizing sequence $\left\{\alpha_{n}\right\}$ for $\theta_{n} \in[0,1]$ and $l_{0}=l$. That is, they used the sequence $\left\{t_{n}\right\}$ given by

$$
\left\{\begin{array}{l}
t_{-1}=0, t_{0}=c, t_{1}=c+\nu \\
t_{n+2}=t_{n+1}+\frac{l\left(t_{n+1}-t_{n-1}\right)\left(t_{n+1}-t_{n}\right)}{1-l\left(t_{n}-t_{n+1}+c\right)}
\end{array}\right.
$$


whereas our sequence $\left\{\alpha_{n}\right\}$ reduces to

$$
\left\{\begin{array}{l}
\alpha_{-1}=0, \alpha_{0}=c, \alpha_{1}=c+\nu \\
\alpha_{n+2}=\alpha_{n+1}+\frac{l\left(\alpha_{n+1}-\alpha_{n-1}\right)\left(\alpha_{n+1}-\alpha_{n}\right)}{1-l_{0}\left(\alpha_{n+1}-\alpha_{n}+c\right)} .
\end{array}\right.
$$

Then, in case $l_{0}<l$ our sequence is more precise (see also (3.17)-(3.19)). Notice also that in the preceding references the sufficient convergence criterion associated to $\left\{t_{n}\right\}$ is given by

$$
l c+2 \sqrt{l \nu} \leq 1
$$

Our sufficient convergence criteria can be also weaker in this case (see also the numerical examples). It is worth nothing that if $c=0$ (3.29) reduces to (3.24) (since $\kappa=2 l$ ).

Similar observations can be made for other choices of parameters.

\section{Local convergence of the secant-type method}

In this section, we present the local convergence analysis of the secant-type method. Let $x^{*} \in \mathcal{X}$ be such that $\mathcal{F}\left(x^{*}\right)=0$ and $\mathcal{F}^{\prime}\left(x^{*}\right)^{-1} \in \mathrm{E}(\mathcal{Y}, \mathcal{X})$. Using the identities

$$
\begin{aligned}
x_{n+1}-x^{*}= & \left(\mathcal{A}_{n}^{-1} \mathcal{F}^{\prime}\left(x^{*}\right)\right) F^{\prime}\left(x^{*}\right)^{-1}\left[\left(\delta \mathcal{F}\left(x_{n}, y_{n}\right)-F^{\prime}\left(x_{n}\right)\right)\right. \\
& \left.+\left(F^{\prime}\left(x_{n}\right)-\delta \mathcal{F}\left(x_{n}, x^{*}\right)\right)\right]\left(x_{n}-x^{*}\right), \\
y_{n}-x_{n}= & \left(1-\theta_{n}\right)\left(x_{n-1}-x_{n}\right),
\end{aligned}
$$

and

$$
y_{n}-x^{*}=\theta_{n}\left(x_{n}-x^{*}\right)+\left(1-\theta_{n}\right)\left(x_{n-1}-x^{*}\right)
$$

we easily arrive at:

Theorem 4.1. Suppose that $\left(\mathcal{D}_{1}\right)$ and $\left(\mathcal{D}_{3}\right)$ hold. Moreover, suppose that there exist $x^{*} \in D, K_{0}>0, K>0$ such that $\mathcal{F}\left(x^{*}\right)=0, F^{\prime}\left(x^{*}\right)^{-1} \in E(\mathcal{Y}, \mathcal{X})$,

$$
\begin{gathered}
\left\|F^{\prime}\left(x^{*}\right)^{-1}\left(\delta \mathcal{F}(x, y)-F^{\prime}\left(x^{*}\right)\right)\right\| \leq K_{0}\left(\left\|x-x^{*}\right\|+\left\|y-x^{*}\right\|\right), \\
\left\|F^{\prime}\left(x^{*}\right)^{-1}\left(\delta \mathcal{F}(x, y)-F^{\prime}(z)\right)\right\| \leq K(\|x-z\|+\|y-z\|) \quad \text { for each } x, y, z \in D,
\end{gathered}
$$
and

$$
\bar{U}\left(x^{*}, R_{0}^{*}\right) \subseteq D
$$

where

$$
R^{*}=\frac{1}{(2 \lambda+1) K+(\lambda+\mu) K_{0}}
$$

and

$$
R_{0}^{*}=(\mu+\lambda-1) R^{*} .
$$

Then the sequence $\left\{x_{n}\right\}$ generated by the secant-type method is well defined, remains in $\bar{U}\left(x^{*}, R^{*}\right)$ for each $n=-1,0,1,2, \ldots$ and converges to $x^{*}$ provided that $x_{-1}, x_{0} \in U\left(x^{*}, R^{*}\right)$. Moreover, the following estimates hold

$$
\left\|x_{n+1}-x^{*}\right\| \leq \hat{e_{n}}\left\|x_{n}-x^{*}\right\| \leq \overline{e_{n}}\left\|x_{n}-x^{*}\right\| \leq e_{n}\left\|x_{n}-x^{*}\right\|,
$$


where

$$
\begin{aligned}
\hat{e_{n}} & =\frac{\bar{K}\left(\left\|x_{n}-x^{*}\right\|+\left|1-\theta_{n}\right|\left\|x_{n-1}-x_{n}\right\|\right.}{1-K_{0}\left(\left[\left(1+\left|\theta_{n}\right|\right)\left\|x_{n}-x^{*}\right\|+\left|1-\theta_{n}\right|\left\|x_{n-1}-x^{*}\right\|\right]\right.}, \\
\overline{e_{n}} & =\frac{\bar{K}\left(\left\|x_{n}-x^{*}\right\|+\lambda\left\|x_{n-1}-x_{n}\right\|\right.}{1-K_{0}\left(\left[\left(\mu\left\|x_{n}-x^{*}\right\|+\lambda\left\|x_{n-1}-x^{*}\right\|\right]\right.\right.}, \\
e_{n} & =\frac{\bar{K}(2 \lambda+1) R^{*}}{1-K_{0}(\lambda+\mu) R^{*}}
\end{aligned}
$$

and

$$
\bar{K}= \begin{cases}\kappa_{0}, & \text { if } n=0, \\ \kappa, & \text { if } n>0 .\end{cases}
$$

Remark 4.2. Comments similar to the one given in Remark 3.3 can also follow for this case. For example, notice again that in the case of Newton's method

$$
R_{*}=\frac{2}{2 \kappa_{0}+\kappa}
$$

whereas the convergence ball given independently by Rheinboldt [17] and Traub [19] is given by

Note that

$$
R_{*}^{1}=\frac{2}{3 \kappa}
$$

$$
R_{*}^{1} \leq R_{*} .
$$

Strict inequality holds in the preceding inequality if $\kappa_{0}<\kappa$. Moreover, the error bounds are tighter, if $\kappa_{0}<\kappa$. Finally, note that $\frac{\kappa_{0}}{\kappa}$ can be arbitrarily small and

$$
\frac{R_{*}}{R_{*}^{1}} \rightarrow 3 \text { as } \frac{\kappa_{0}}{\kappa} \rightarrow 0
$$

\section{Numerical examples}

Related to the semilocal case we present the following examples.

Example 5.1. Let $\mathcal{X}=\mathcal{Y}=\mathbb{R}$ and let consider the following function

$$
x^{3}-0.49=0,
$$

and we are going to apply the secant method $\left(\lambda=1, \mu=1, \theta_{n}=0\right)$ to find the solution of (5.1). We take the starting points $x_{-1}=1.14216 \cdots, x_{0}=1$ and we consider the domain $\Omega=B\left(x_{0}, 2\right)$. In this case, we obtain

$$
\begin{gathered}
\nu=0.147967 \cdots, \\
\nu=0.14216 \cdots, \\
l=2.61119 \cdots, \\
l_{0}=1.74079 \cdots .
\end{gathered}
$$


Notice that hypothesis $l c+2 \sqrt{l \nu} \leq 1$ is not satisfied, but hypotheses of Theorem 3.2 are satisfied, so the convergence of secant method starting form $x_{0} \in B\left(x_{0}, 2\right)$ converges to the solution of (5.1).

Example 5.2. Let $\mathcal{X}=\mathcal{Y}=\mathcal{C}[0,1]$, equipped with the max-norm. Consider the following nonlinear boundary value problem

$$
\left\{\begin{aligned}
u^{\prime \prime} & =-u^{3}-\gamma u^{2} \\
u(0) & =0, \quad u(1)=1
\end{aligned}\right.
$$

It is well known that this problem can be formulated as the integral equation

$$
u(s)=s+\int_{0}^{1} \mathcal{Q}(s, t)\left(u^{3}(t)+\gamma u^{2}(t)\right) d t,
$$

where $\mathcal{Q}$ is the Green's function:

$$
\mathcal{Q}(s, t)=\left\{\begin{array}{cc}
t(1-s), & t \leq s \\
s(1-t), & s<t .
\end{array}\right.
$$

We observe that

$$
\max _{0 \leq s \leq 1} \int_{0}^{1}|\mathcal{Q}(s, t)| d t=\frac{1}{8}
$$

Then the problem (5.6) is in the form (1.1), where $F: \mathcal{D} \longrightarrow \mathcal{Y}$ is defined as

$$
[F(x)](s)=x(s)-s-\int_{0}^{1} \mathcal{Q}(s, t)\left(x^{3}(t)+\gamma x^{2}(t)\right) d t .
$$

We define the divided difference by $\delta F(x, y)=\int_{0}^{1} F^{\prime}(y+t(x-y)) d t$. Set $u_{0}(s)=s$ and $\mathcal{D}=U\left(u_{0}, R_{0}\right)$. It is easy to verify that $U\left(u_{0}, R_{0}\right) \subset U\left(0, R_{0}+1\right)$ since $\left\|u_{0}\right\|=1$. If $2 \gamma<5$, the operator $F^{\prime}$ satisfies conditions of Theorem 3.2 , with

$\theta_{n}=0, \nu=\frac{1+\gamma}{\left(1-l_{0} c\right)(5-2 \gamma)}, l=\frac{\gamma+6 R_{0}+3}{\left(1-l_{0} c\right)(5-2 \gamma)}, l_{0}=\frac{2 \gamma+3 R_{0}+6}{\left(1-l_{0} c\right)(5-2 \gamma)}$.

Since

$$
\begin{aligned}
\left\|\delta F\left(x_{0}, x_{-1}\right)^{-1} F\left(x_{0}\right)\right\| & \leq\left\|\delta F\left(x_{0}, x_{-1}\right)^{-1} F^{\prime}\left(x_{0}\right)\right\|\left\|F^{\prime}\left(x_{0}\right) F\left(x_{0}\right)\right\| \\
& \leq \frac{1}{\left(1-l_{0} c\right)} \frac{1+\gamma}{5-2 \gamma} .
\end{aligned}
$$

Note that $l_{0}<l$. Therefore, the hypothesis of Kantorovich may not be satisfied, but conditions of Theorem 3.2 may be satisfied.

Finally, for the local case we study the following one.

Example 5.3. Let $X=Y=\mathbb{R}^{3}, D=U(0,1), x^{*}=(0,0,0)$ and define function $F$ on $D$ by

$$
F(x, y, z)=\left(e^{x}-1, y^{2}+y, z\right)
$$


We have that for $u=(x, y, z)$

$$
F^{\prime}(u)=\left(\begin{array}{ccc}
e^{x} & 0 & 0 \\
0 & 2 y+1 & 0 \\
0 & 0 & 1
\end{array}\right) .
$$

Using the norm of the maximum of the rows and (5.7)-(5.8) we see that since $F^{\prime}\left(x^{*}\right)=\operatorname{diag}\{1,1,1\}$, we can define parameters for Newton's method by

$$
\begin{gathered}
K=e / 2, \\
K_{0}=1, \\
R^{*}=\frac{2}{e+4}, \\
R_{0}^{*}=R^{*},
\end{gathered}
$$

since $\theta_{n}=1, \mu=2, \lambda=0$. Then the Newton's method starting form $x_{0} \in$ $B\left(x^{*}, R^{*}\right)$ converges to a solution of (5.7). Note that using only Lipschitz condition we obtain the Rheinboldt or Traub ball $R_{T R}^{*}=\frac{2}{3 e}<R^{*}$.

Example 5.4. In this example we present an application of the previous analysis to the Chandrasekhar equation:

$$
x(s)=1+\frac{s}{4} x(s) \int_{0}^{1} \frac{x(t)}{s+t} d t, \quad s \in[0,1],
$$

which arises in the theory of radiative transfer $[6] ; x(s)$ is the unknown function which is sought in $C[0,1]$. The physical background of this equation is fairly elaborate. It was developed by Chandraseckhar [6] to solve the problem of determination of the angular distribution of the radiant flux emerging from a plane radiation field. This radiation field must be isotropic at a point, that is the distribution in independent of direction at that point. Explicit definitions of these terms may be found in the literature [6]. It is considered to be the prototype of the equation,

$$
x(s)=1+\lambda s x(s) \int_{0}^{1} \frac{\varphi(s)}{s+t} x(t) d t, \quad s \in[0,1],
$$

for more general laws of scattering, where $\varphi(s)$ is an even polynomial in $s$ with

$$
\int_{0}^{1} \varphi(s) d s \leq \frac{1}{2} .
$$

Integral equations of the above form also arise in the other studies [6]. We determine where a solution is located, along with its region of uniqueness.

Note that solving (3.7) is equivalent to solve $F(x)=0$, where $F: C[0,1] \rightarrow$ $C[0,1]$ and

$$
[F(x)](s)=x(s)-1-\frac{s}{4} x(s) \int_{0}^{1} \frac{x(t)}{s+t} d t, \quad s \in[0,1] .
$$


To obtain a numerical solution of (3.7), we first discretize the problem and approach the integral by a Gauss-Legendre numerical quadrature with eight nodes,

$$
\int_{0}^{1} f(t) d t \approx \sum_{j=1}^{8} w_{j} f\left(t_{j}\right)
$$

where

$$
\begin{aligned}
& t_{1}=0.019855072, t_{2}=0.101666761, t_{3}=0.237233795, t_{4}=0.408282679 \\
& t_{5}=0.591717321, t_{6}=0.762766205, t_{7}=0.898333239, t_{8}=0.980144928 \\
& w_{1}=0.050614268, w_{2}=0.111190517, w_{3}=0.156853323, w_{4}=0.181341892 \\
& w_{5}=0.181341892, w_{6}=0.156853323, w_{7}=0.111190517, w_{8}=0.050614268
\end{aligned}
$$

TABLE 1. The comparison results of $\left\|\bar{x}_{n+1}-\bar{x}_{n}\right\|$ for Example 3.3 using various methods.

\begin{tabular}{ccccc}
\hline$n$ & $\left\|x_{n+1}-x_{n}\right\|$ & $\left\|x_{n+1}-x_{n}\right\|$ & $\left\|x_{n+1}-x_{n}\right\|$ & $\left\|x_{n+1}-x_{n}\right\|$ \\
& $\theta_{n}=0$, Newton & $\theta_{n}=1$, secant & $\theta_{n}=2$, Kurchatov & $\theta_{n}=1 / 2$, midpoint \\
\hline 1 & $9.49639 \times 10^{-6}$ & $4.70208 \times 10^{-2}$ & $4.33999 \times 10^{-1}$ & $1.42649 \times 10^{-1}$ \\
2 & $8.18823 \times 10^{-12}$ & $7.77292 \times 10^{-3}$ & $3.28371 \times 10^{-2}$ & $1.51900 \times 10^{-2}$ \\
3 & $5.15077 \times 10^{-24}$ & $5.14596 \times 10^{-5}$ & $2.33370 \times 10^{-3}$ & $1.66883 \times 10^{-4}$ \\
4 & $1.79066 \times 10^{-48}$ & $3.89016 \times 10^{-8}$ & $9.32850 \times 10^{-6}$ & $1.34477 \times 10^{-7}$ \\
5 & $1.95051 \times 10^{-97}$ & $1.77146 \times 10^{-13}$ & $2.214411 \times 10^{-9}$ & $1.03094 \times 10^{-12}$ \\
6 & $2.12404 \times 10^{-195}$ & $5.35306 \times 10^{-22}$ & $1.801201 \times 10^{-15}$ & $5.63911 \times 10^{-21}$ \\
\hline$\rho$ & 2.00032 & 1.61815 & 1.61854 & 1.61817 \\
\hline
\end{tabular}

If we denote $x_{i}=x\left(t_{i}\right), i=1,2, \ldots, 8$, the equation (3.7) is transformed into the following nonlinear system:

$$
x_{i}=1+\frac{x_{i}}{4} \sum_{j=1}^{8} a_{i j} x_{j}, \quad i=1,2, \ldots, 8,
$$

where, $a_{i j}=\frac{t_{i} w_{j}}{t_{i}+t_{j}}$.

Denote now $\bar{x}=\left(x_{1}, x_{2}, \ldots, x_{8}\right)^{T}, \overline{1}=(1,1, \ldots, 1)^{T}, A=\left(a_{i j}\right)$ and write the last nonlinear system in the matrix form:

$$
\bar{x}=\overline{1}+\frac{1}{4} \bar{x} \odot(A \bar{x}),
$$

where $\odot$ represents the inner product. Set $G(x)=x$. If we choose $\bar{x}_{0}=$ $(1,1, \ldots, 1)^{T}$ and $\bar{x}_{-1}=(0,0, \ldots, 0)^{T}$. Assume sequence $\left\{\bar{x}_{n}\right\}$ is generated by secant-type methods with different choices of $\theta_{n}$. Table 1 gives the comparison results for $\left\|\bar{x}_{n+1}-\bar{x}_{n}\right\|$ equipped with the max-norm for this example. The computational order of convergence (COC) is shown in Table 1 for various methods. Here (COC) is defined in [1], [3] by

$$
\rho \approx \ln \left(\frac{\left\|\bar{x}_{n+1}-\bar{x}^{\star}\right\|_{\infty}}{\left\|\bar{x}_{n}-\bar{x}^{\star}\right\|_{\infty}}\right) / \ln \left(\frac{\left\|\bar{x}_{n}-\bar{x}^{\star}\right\|_{\infty}}{\left\|\bar{x}_{n-1}-\bar{x}^{\star}\right\|_{\infty}}\right), \quad n \in \mathbb{N}
$$


The last line in Table 1 shows the (COC).

\section{References}

[1] S. Amat, S. Busquier, and M. Negra, Adaptive approximation of nonlinear operators, Numer. Funct. Anal. Optim. 25 (2004), no. 5-6, 397-405.

[2] I. K. Argyros, Computational Theory of Iterative Methods, Studies in Computational Mathematics, 15. Elsevier B. V., Amsterdam, 2007.

[3] I. K. Argyros, Y. J. Cho, and S. Hilout, Numerical Method for Equations and Its Applications, CRC Press/Taylor and Francis, New York, 2012.

[4] I. K. Argyros and S. Hilout, Weaker conditions for the convergence of Newton's method, J. Complexity 28 (2012), no. 3, 364-387.

[5] E. Cătinaş, The inexact, inexact perturbed, and quasi-Newton methods are equivalent models, Math. Comp. 74 (2005), no. 249, 291-301.

[6] S. Chandrasekhar, Radiative Transfer, Dover Publ., New York, 1960.

[7] J. E. Dennis, Toward a unified convergence theory for Newton-like methods, Nonlinear Functional Anal. and Appl. (Proc. Advanced Sem., Math. Res. Center, Univ. of Wisconsin, Madison, Wis., 1970) pp. 425-472 Academic Press, New York, 1971.

[8] J. A. Ezquerro, J. M. Gutiérrez, M. A. Hernández, N. Romero, and M. J. Rubio, The Newton method: from Newton to Kantorovich, (Spanish), Gac. R. Soc. Mat. Esp. 13 (2010), no. 1, 53-76.

[9] W. B. Gragg and R. A. Tapia, Optimal error bounds for the Newton-Kantorovich theorem, SIAM J. Numer. Anal. 11 (1974), 10-13.

[10] M. A. Hernández, M. J. Rubio, and J. A. Ezquerro, Secant-like methods for solving nonlinear integral equations of the Hammerstein type, J. Comput. Appl. Math. 115 (2000), no. 1-2, 245-254.

[11] L. V. Kantorovich and G. P. Akilov, Functional Analysis, Pergamon Press, Oxford, 1982.

[12] Á. A. Magreñán, Estudio de la dinámica del método de Newton amortiguado, PhD Thesis, Servicio de Publicaciones, Universidad de La Rioja, 2013.

[13] L. M. Ortega and W. C. Rheinboldt, Iterative Solution of Nonlinear Equations in Several Variables, Academic press, New York, 1970.

[14] F. A. Potra and V. Pták, Nondiscrete induction and iterative processes, Research Notes in Mathematics, 103. Pitman (Advanced Publishing Program), Boston, MA, 1984.

[15] P. D. Proinov, General local convergence theory for a class of iterative processes and its applications to Newton's method, J. Complexity 25 (2009), no. 1, 38-62.

[16] - New general convergence theory for iterative processes and its applications to Newton-Kantorovich type theorems, J. Complexity 26 (2010), no. 1, 3-42.

[17] W. C. Rheinboldt, An adaptive continuation process for solving systems of nonlinear equations, Mathematical models and numerical methods (Papers, Fifth Semester, Stefan Banach Internat. Math. Center, Warsaw, 1975), pp. 129-142, Banach Center Publ., 3, PWN, Warsaw, 1978.

[18] J. W. Schmidt, Untere Fehlerschranken für Regula-Falsi Verhafren, Period. Math. Hungar. 9 (1978), no. 3, 241-247.

[19] J. F. Traub, Iterative Methods for the Solutions of Equations, Prentice-Hall, New Jersey, 1964.

[20] T. Yamamoto, A convergence theorem for Newton-like methods in Banach spaces, Numer. Math. 51 (1987), no. 5, 545-557.

[21] P. P. Zabrejko and D. F. Nguen, The majorant method in the theory of NewtonKantorovich approximations and the Pták error estimates, Numer. Funct. Anal. Optim. 9 (1987), no. 5-6, 671-684. 
IOANNis Konstantinos ARgyros

Cameron University

Department of Mathematics Sciences

LAWTON, OK 73505, USA

E-mail address: iargyros@cameron.edu

Ángel Alberto Magreñán

UNIVERSIDAD INTERNACIONAL DE LA RIOJA (UNIR)

Departamento de TFG/TFM

26002 Logroño, LA RioJA, SPAIN

E-mail address: angel.magrenan@unir.net 